\title{
Experimental search for long-range forces in neutron scattering via a gravitational spectrometer
}

\author{
A. P. Serebrov, ${ }^{1,}{ }^{*}$ P. Geltenbort, ${ }^{2}$ O. M. Zherebtsov, ${ }^{1}$ S. V. Sbitnev, ${ }^{1}$ V. E. Varlamov, ${ }^{1}$ A. V. Vassiljev, ${ }^{1}$ M. S. Lasakov, ${ }^{1}$ \\ I. A. Krasnoschekova, ${ }^{1}$ S. N. Ivanov, ${ }^{2}$ and D. Pushin ${ }^{3}$ \\ ${ }^{1}$ Petersburg Nuclear Physics Institute, 188300 Gatchina, Leningrad District, Russia \\ ${ }^{2}$ Institute Laue Langevin, BP 156, F-38042 Grenoble, France \\ ${ }^{3}$ University of Waterloo, Ontario, Canada and National Institute of Standards and Technology, Gaithersburg, Maryland 20899, USA
}

(Received 24 June 2013; revised manuscript received 19 November 2013; published 9 April 2014)

\begin{abstract}
In this work we introduce a method of measuring low-energy scattering cross section with a gravitational spectrometer. In this method we add atoms (i.e., He) to the gravitational spectrometer filled with a target gas of ultracold neutrons (UCN). We search for long-range forces between atoms and UCN by measuring transfer of a small recoil energy $\sim 10^{-7} \mathrm{eV}$ using the gravitational spectrometer. As a result of this search we set new constraints on the strength of long-range forces within the range of the effective radius of interaction of $10^{-7}-10^{-4} \mathrm{~cm}$.
\end{abstract}

DOI: 10.1103/PhysRevC.89.044002

PACS number(s): 29.90.+r, 28.20.-v, 04.80.-y, 14.80.-j

\section{INTRODUCTION}

Identifying deviations of gravitational interaction from the $1 / r^{2}$ law (inverse-square law) in the range of small distances is extremely important to verify theories predicting the presence of additional dimensions [1,2] and supersymmetric theories predicting the existence of new very light particles. The exchange of these particles results in additional interactions between nucleons [3-5]. A survey of theoretical and experimental works on the search for deviations from the inverse-square law is presented in [6,7].

There are different methods of identifying long-range forces in the interaction of elementary particles [6-8]. Within the range of $10^{-11}-10^{-9} \mathrm{~cm}$ investigations are carried out using neutrons at the energies of the order of electron volts $[9,10]$. Laboratory experiments on gravitational interactions of bodies have been performed for distances $10^{-4}-10^{-2} \mathrm{~cm}$ [11-20]. There are somewhat effective methods using thermal and cold neutrons within the range of $10^{-10}-10^{-4} \mathrm{~cm}[9,21]$.

The scattering amplitude of a neutron scattered by atoms can be expressed as follows:

$$
f(q)=f_{\text {nucl }}+f_{n-e}(q)+f_{\text {long range }}(q),
$$

where $f_{\text {nucl }}$ is a nuclear scattering amplitude usually expressed through scattering length $b$, with $f_{\text {nucl }}=-b . f_{n-e}(q)$ is the neutron-electron scattering amplitude from neutron scattering by charges distributed inside the nucleus and the electron shell of atoms. Furthermore, we will not consider contribution from the $n-e$ interaction, because this effect occurs mainly for fast neutrons [22]. The last term in Eq. (1) relates to a hypothetical long-range interaction (compared to the nuclear one) of a neutron with a nucleus. $f_{\text {long range }}(q)$ is a spin-independent amplitude of interaction likely to arise as a result of exchange by a scalar or vector boson. In the case of a scalar boson, the interaction potential is an attractive one, while for a vector boson exchange the interaction potential is a repulsive one:

$$
\varphi(r)=\frac{ \pm g_{ \pm}^{2} M \hbar c e^{-r / \lambda}}{4 \pi r},
$$

*serebrov@pnpi.spb.ru where $M$ is the mass of the interaction particle expressed in units of nucleon mass $m_{n}$, with $\lambda$ being an effective radius of interaction and $g_{ \pm}^{2}$ being a dimensionless coupling constant. It should be noted that in the general case $M=m_{1} m_{2} / m_{n}^{2}$, with $m_{1}, m_{2}$ being masses of interaction particles. In our consideration we have assumed a proton and a neutron forming the atom nucleus to interact similarly with a free neutron through long-range forces. We have also assumed that electrons do not make a contribution to the interaction of a free neutron and atom. In Eq. (2) the upper sign corresponds to a vector-type interaction, whereas the lower sign corresponds to a scalar-type interaction.

In a similar way, the amplitude within the Bohr approximation can be presented as follows:

$$
\begin{aligned}
f_{\text {long_range }}(q) & =-\frac{m}{2 \pi \hbar^{2}} \int \varphi(r) e^{-i \vec{q} \vec{r}} d V \\
& =\frac{\mp 2 m}{\hbar^{2}} \frac{g_{ \pm}^{2} M \hbar c}{4 \pi} \frac{\lambda^{2}}{(\lambda q)^{2}+1},
\end{aligned}
$$

where $m$ is a reduced mass $m=\frac{m_{n} m_{A}}{m_{n}+m_{A}}$, mass of a gas atom $m_{A}=m_{n} M, q=\left|\vec{k}^{\prime}-\vec{k}\right|$ is a momentum transferred to a neutron, and $\vec{k}$ and $\vec{k}^{\prime}$ are wave vectors of the particle in the center of mass before and after collision.

An experimental search for additional terms in the scattering amplitude can be based on the fact that a long-range interaction makes a contribution to the scattering amplitude either at a small transferred momentum $q$ or at small scattering angles. The scattering amplitude at $\theta=0$ or $q=0$ can be measured with a high accuracy in neutron-optical experiments by a perfect crystal neutron interferometer [21]. This result should be compared to $f_{\text {nucl }}=-b$ to find the presence of additional terms in Eq. (1). For example, comparison of interferometer measurements with the Bragg diffractometer data imposes rigid constraint on $f_{\text {long range }}(q)$ [9].

A small angle scattering method would be a direct way of measuring $f_{\text {long range }}(q)$, as existence of long-range forces gives rise to scattering at small angles. This method has some obvious limitations because of the existence of small angle scattering caused by sample roughness as well as multiple scattering. It is to be noted that initial divergence of a beam 
does not permit scattering to be distinguished at very small angles from the beam divergence.

In the previous work [23] we introduced a different approach to small angle scattering that measures small recoil energies rather than small scattering angles. This approach uses UCN gas as a scattering target for a flux of atoms introduced to the same trap. For thermal neutrons the scattering angle corresponding to recoil energy of $\sim 10^{-7} \mathrm{eV}$ is $2 \mathrm{mrad}$, which is within the divergence of the incident neutron beam. For cold neutrons this scattering angle is twice the value, however, it does not exceed the divergence of the neutron beam either.

Furthermore, the previous work [23] compares scattering cross sections of $\mathrm{He}$ atoms obtained employing three different methods: the neutron interferometer method, the neutron transmission method, and the UCN storage time method. The value $b_{\text {bound_int }}$ (bound coherent scattering length) is taken from [24] $\left(b_{\text {bound_int }}(\mathrm{He})=3.26 \pm 0.03 \mathrm{fm}\right)$. Because the UCN storage time method measures neutron scattering with free nuclei, the scattering length should be recalculated using the equation $b_{\text {free_int }}(\mathrm{He})=b_{\text {bound_int }}(\mathrm{He}) \times M_{\mathrm{He}} /\left(M_{\mathrm{He}}+1\right)=$ $0.2604(24) \times 10^{-12} \mathrm{~cm}$, where $M_{\mathrm{He}}$ is the ratio of the $\mathrm{He}$ atom to neutron mass. Accordingly, the recalculated cross section will be $\sigma_{\mathrm{coh}}(\mathrm{He})=4 \pi b^{2}(\mathrm{He})_{\text {free_int }}=0.852(16) \times 10^{-24} \mathrm{~cm}^{2}$. The scattering cross section $\sigma_{\text {free_nucl }}(\mathrm{He})$ measured by the neutron transmission method is $(0.773 \pm 0.009) \times 10^{-24} \mathrm{~cm}^{2}$ (for neutron energies ranging from 0.19 to $6.19 \mathrm{eV}$ ) [25]. The neutron cross-section data tables from [26] show the assumed average value to be $(0.76 \pm 0.01) \times 10^{-24} \mathrm{~cm}^{2}$, being five standard deviations from interferometry results. The scattering cross section measured by the UCN method $[23,27]$ has the value of $(0.740 \pm 0.006) \times 10^{-24} \mathrm{~cm}^{2}$, being six standard deviations from the assumed average value given in [28] for the cross section derived from the coherent scattering length for helium. The effect involved can be caused either by the existence of long-range forces or systematic experimental errors.

This article presents results of an experimental search for long-range forces previously proposed in [23]. The measurement of the above-barrier neutron flux scattered by a noble gas of He atoms was carried out. The aim of this paper is to search for evidence of small amounts of energy transferred to UCN from the long-range forces. Figure 1 shows the differential cross section produced by long-range forces versus transferred energies. For example, at $\lambda=10^{-6} \mathrm{~cm}, \mathrm{~g}^{2}=2 \times 10^{-14}$, with transferred energy being $\sim 10^{-7} \mathrm{eV}$, the differential cross section produced by long-range forces is six times higher than the nuclear one, so its contribution to a total cross section is not negligible.

\section{EXPERIMENTAL SETUP}

The experiment was carried out using the PF2 beam of the UCN facilities of the ILL reactor. The scheme of the experimental setup is shown in Fig. 2. The apparatus consists of a UCN spectrometer with filling and emptying valves, the ultracold neutron guide system, UCN detectors, and the vacuum system. The UCN spectrometer is a vertical cylinder with a diameter of $60 \mathrm{~cm}$ and height of $200 \mathrm{~cm}$. It is made of copper with the inner surface coated with beryllium. The critical velocity of this coating is $6.8 \mathrm{~m} / \mathrm{s}$ (i.e., velocity of

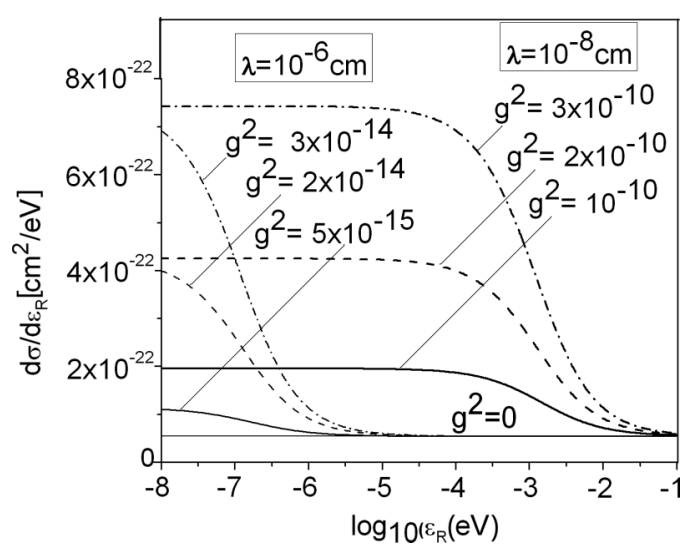

FIG. 1. Dependence of differential cross section on recoil energy $\varepsilon_{R}$ transferred to a neutron for various values of parameter $\lambda$ for the case of a repulsive potential.

neutrons has to be above this critical velocity for them to escape from the UCN spectrometer).

The UCN fill the trap (2) while the valve (1) is open, with valves (4) and (6) being closed. The absorber (3) inside the trap is placed in its bottom position at a certain distance " $h$ " from the bottom of the trap. As soon as the equilibrium density in the trap is achieved, the valve (1) is closed. The foil at critical energy of the UCN, with $E=m_{n} g h(1 \mathrm{neV}=1.02 \mathrm{~cm})$, is placed behind the exit valve (4) (where $m_{n}$ is a neutron mass and $g$ is free-fall acceleration). This foil serves to analyze the spectrum of the UCN in emptying the trap. The UCN are stored in the trap for a holding time $t$ to form a pure spectrum at peak UCN energy of $m_{n} g h$. Then the absorber (3) is moved to its upper position at the top of the trap. After obtaining low recoil energy, the UCN can overcome the potential barrier of the foil (8) at the exit guide and can be registered by the detector (5). UCN are counted immediately after the absorber

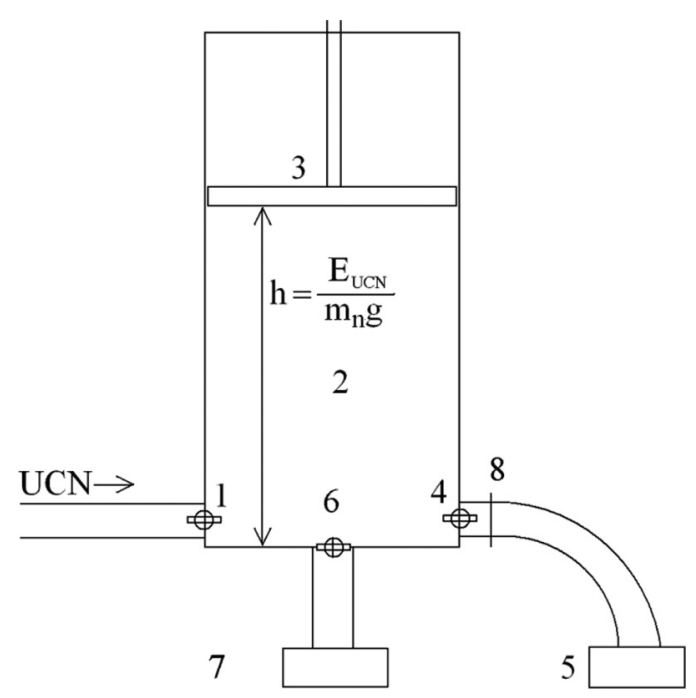

FIG. 2. Experimental setup 1, entrance valve; 2, UCN trap with critical energy $E_{\mathrm{UCN}}^{\mathrm{trap}} ; 3$, absorber for formation of UCN spectrum; 4, exit valve; 5, UCN detector A; 6, exit valve; 7, UCN detector B; 8 , foil. 
(3) is moved to its top position and the valve (4) is opened. The background of detector (5) is measured with valve (4) closed. Both valve (6) and detector (7) are used for counting $\mathrm{UCN}$ in the spectrometer. To distinguish between quasielastic scattering of UCN on the surface of the trap and that of noble gas atoms the measurements are made with noble gas in the trap as well as under high vacuum. Using such a measurement scheme it is possible to determine with high precision an extremely low energy transfer to UCN from the introduced gas.

A foil made of zirconium (8), as thick as $10 \mu \mathrm{m}$, is employed to create a gravitational barrier for UCN. The $99.8 \%$ purity foil is produced by the Goodfellow company.

Helium gas used in our experiment is produced by Messer. The $99.95 \%$ purity gas is kept in a vessel at high pressure. The delivery gas system is made up of a fine adjustment leakage, a delivery pipe, and lock valves.

The pressure of He gas inside the spectrometer is measured with an absolute pressure sensor (CERAVAC CTR 100) made by Leybold. The sensor has an upper pressure limit of 10 Torr and the measurement error is between 0.002 and 0.02 Torr (depending on the measured pressure).

An experimental hall of the ILL reactor (D level) is equipped with air temperature control and a stabilization system. The temperature readings are accessible to us.

\section{EXPERIMENTAL TECHNIQUE}

The experimental task is to register UCN transferred from 0 to $E_{\mathrm{UCN}}^{\text {foil }}=m_{n} g h$ energy range into the energy range from $E_{\mathrm{UCN}}^{\text {foil }}$ to $E_{\mathrm{UCN}}^{\text {trap }}$, i.e., from subbarrier neutrons to above-barrier ones. The number of initial neutrons $N_{\mathrm{UCN}}^{\text {spect }}$ in the spectrometer changes with time according to the equation:

$$
N_{\mathrm{UCN}}^{\text {spect }}(t)=N_{0}^{\text {spect }} e^{-t / \tau_{\text {stor below }}}
$$

with $N_{0}^{\text {spect }}$ being the number of sub-barrier UCN in the spectrometer at the moment of moving the absorber (3) to its top position, and $\tau_{\text {storbelow }}$ being the UCN storage time in the spectrometer with energy lower than boundary energy of the foil $E_{\mathrm{UCN}}^{\text {foil }}=76 \mathrm{neV}$.

The number of the so-called above-barrier neutrons $N_{\mathrm{up}}^{\text {spect }}$, i.e., neutrons in the energy range from $E_{\mathrm{UCN}}^{\text {foil }}$ to $E_{\mathrm{UCN}}^{\text {trap }}$, is determined by their emergence from the 0 to $E_{\mathrm{UCN}}^{\text {foil }}$ energy range with the probability of $W^{\text {up }}$, by their losses in the trap with the probability of $\tau_{\text {stor up }}^{-1}$, as well as by their leakage out of the trap with the probability of $\tau_{\text {emp f }}^{-1}$. The total probability of above-barrier UCN being lost is $\tau_{\text {tot up }}^{-1}=\tau_{\text {stor up }}^{-1}+\tau_{\text {emp f }}^{-1}$.

Correlation between these two processes can be described by an inhomogeneous differential equation:

$$
\frac{d N_{\mathrm{up}}^{\mathrm{spect}}(t)}{d t}=-\tau_{\mathrm{totup}}^{-1} N_{\mathrm{up}}^{\mathrm{spect}}(t)+W^{\mathrm{up}} N_{0} e^{-t / \tau_{\text {sto below }}} .
$$

The solution to this equation under initial condition $N_{\text {up }}^{\text {spect }}(0)=0$ is as follows:

$$
\begin{aligned}
N_{\text {up }}^{\text {spect }}(t)= & N_{0}^{\text {spect }} W^{\text {up }} \frac{1}{\tau_{\text {tot up }}^{-1}-\tau_{\text {stor below }}^{-1}} \\
& \times\left(e^{-t / \tau_{\text {stor below }}}-e^{-t / \tau_{\text {tot up }}}\right) .
\end{aligned}
$$

It should be noted that Eq. (6) is composed of two different UCN storage times $\tau_{\text {storbelow }}$ and $\tau_{\text {stor up }}$, as UCN storage time in the trap is dependent on UCN energy. In addition, at the open shutter (4) probability of above-barrier neutron storage is determined not only by probability of losses on the trap walls $\tau_{\text {stor up }}^{-1}$ but also by probability of leakage of above-barrier $\mathrm{UCN}$ onto the detector $\tau_{\text {emp f }}^{-1}$, hence, $\tau_{\text {tot up }}^{-1}=\tau_{\text {stor up }}^{-1}+\tau_{\text {emp f }}^{-1}$.

Equation (6) describes a dynamic process of accumulating and subsequent decrease of above-barrier neutrons in the spectrometer. This process can be registered with detector A (5). However, for calculating the count rate of above-barrier neutrons with detector $d N_{\mathrm{up}}^{\mathrm{det}} / d t$ one should take account of the probability of UCN leakage onto the detector $\tau_{\text {emp }}^{-1}$, the factor of UCN losses in foil $k_{f}$, and detector efficiency $\varepsilon$,

$$
\frac{d N_{\mathrm{up}}^{\mathrm{det}}}{d t}=\tau_{\mathrm{empf}}^{-1} \varepsilon k_{f} N_{\mathrm{up}}^{\mathrm{spec}}(t) .
$$

After integrating by time it follows that during a complete measuring cycle from zero to infinity the detector would register the given number of above-barrier $\mathrm{UCN}$ :

$$
N_{\text {up }}^{\text {det }}=N_{0}^{\text {spect }} \tau_{\text {stor below }} W^{\text {up }} k_{f} \frac{\tau_{\text {emp f }}^{-1}}{\tau_{\text {stor up }}^{-1}+\tau_{\text {emp f }}^{-1}} .
$$

This equation can be easily interpreted. The terms $N_{0}^{\text {spect }} \tau_{\text {stor below }} W^{\text {up }}$ determine the total number of above-barrier neutrons which have emerged during the entire process from zero to infinity. The multiplier $\tau_{\text {emp f }}^{-1} /\left(\tau_{\text {stor up }}^{-1}+\tau_{\text {emp f }}^{-1}\right)$ determines the neutron fraction to be recorded with a detector, i.e., the ratio of leakage probability of above-barrier neutrons through the foil to the total probability for above-barrier neutrons. Finally, the coefficient $k_{f}$ takes account of losses of above-barrier UCN inside the foil. The efficiency of abovebarrier UCN detection with a detector at $100 \%$ efficiency can be calculated as follows:

$$
P_{\text {up }}^{\text {det }}=k_{f} \frac{\tau_{\text {empf }}^{-1}}{\tau_{\text {stor up }}^{-1}+\tau_{\text {emp f }}^{-1}} .
$$

However, Eq. (8) contains the value of $N_{0}^{\text {spect }}$ to be measured experimentally. The conventional procedure of making measurements is to open the exit valve (4) at the time $t=0$ (after formation of the sub-barrier neutron spectrum with an absorber) and to cause sub-barrier neutrons to leak onto the detector without foil (8). The number of neutrons recorded with the detector during the process will be as follows:

$$
N_{0}^{\text {det }} \text { below }=N_{0}^{\text {spect }} \frac{\tau_{\text {emp below }}^{-1}}{\tau_{\text {stor below }}^{-1}+\tau_{\text {emp below }}^{-1}} \varepsilon,
$$

where $\tau_{\text {emp below }}^{-1}$ is the probability of leakage of sub-barrier neutrons onto the detector without foil. Hence, the efficiency of detecting sub-barrier neutrons by the installation with the detector at $100 \%$ efficiency will be as follows:

$$
P_{\text {below }}^{\text {det }}=\frac{\tau_{\text {emp below }}^{-1}}{\tau_{\text {stor below }}^{-1}+\tau_{\text {emp below }}^{-1}} .
$$


Thus, to measure the probability of transfer of sub-barrier neutrons into above-barrier ones the following relation is used:

$$
W^{\text {up }}=\frac{N_{\text {up }}^{\text {det }}}{N_{0}^{\text {det }} \text { below }} \frac{P_{\text {below }}^{\text {det }}}{P_{\text {up }}^{\text {det }}} \frac{1}{\tau_{\text {stor below }}} .
$$

It is to be noted that the detector efficiency $\varepsilon$ reduces this ratio.

Consequently, to determine the value $W^{\text {up }}$ to be obtained (probability of low heating) one must measure a number of values included in Eq. (12).

(1.) $N_{\mathrm{up}}^{\mathrm{det}}$, an integral number of above-barrier neutrons registered with detector A during a long period of storing sub-barrier neutrons in the trap. Measurements are made with foil.

(2.) $N_{0}^{\text {det }}$ below, an integral number of sub-barrier neutrons escaped from the trap after forming the spectrum and opening the valve (4). Measurements are made with detector A without foil.

(3.) $\tau_{\text {storbelow }}$, storage time of sub-barrier neutrons. Measurements are made either with detector B or detector A without foil. It is also possible to measure $\tau_{\text {stor below }}$ with detector B directly during storage of sub-barrier UCN in the trap, as a small leak valve closure (6) allows measuring of the UCN density decrease during storage.

(4.) $P_{\text {below }}^{\text {det }}$, efficiency of registering sub-barrier neutrons by the installation. According to Eq. (11) it is required that the sub-barrier neutron leakage time from the trap $\left(\tau_{\text {stor below }}^{-1}+\tau_{\text {emp below }}^{-1}\right)^{-1}$ should be measured and the above discussed value $\tau_{\text {stor below }}$ be used. Measurements of leakage time of sub-barrier neutrons are made without foil.

(5.) $P_{\text {up }}^{\text {det }}$, efficiency of registering sub-barrier neutrons by the installation with a detector at $\varepsilon=100 \%$. Direct use of Eq. (9) and measurement of $\tau_{\text {emp }}$ is of considerable difficulty. Above-barrier neutrons at energy close to the boundary energy of the foil have a very small probability of leakage. Measuring of corresponding times becomes unreasonable. Thus, the registration efficiency of above-barrier neutrons can be determined by means of an experimental coefficient of abovebarrier neutron transmission through the foil:

$$
T_{\text {foil }}(E)=\frac{N_{A}^{f}(E)}{N_{A}(E)},
$$

where $N_{A}^{f}(E)$ is an energy spectrum of UCN leakage onto detector A from the spectrometer through the foil, and $N_{A}(E)$ is an energy spectrum of UCN leakage directly onto detector A. Transmission coefficient $T_{\text {foil }}(E)$ already includes UCN losses inside the foil. However, to take account of above-barrier UCN losses, one must use multiplier $\tau_{\text {emp up }}^{-1} /\left(\tau_{\text {stor up }}^{-1}+\tau_{\text {emp up }}^{-1}\right)$ :

$$
P_{\text {up }}^{\text {det }}(E)=T_{\text {foil }}(E) \frac{\tau_{\text {emp up }}^{-1}}{\tau_{\text {stor up }}^{-1}+\tau_{\text {emp up }}^{-1}},
$$

where $\tau_{\text {emp up }}^{-1}$ is the leakage probability of above-barrier neutrons in the experiment without foil, $\left(\tau_{\text {stor up }}^{-1}+\tau_{\text {empup }}^{-1}\right)=$ $\tau_{\text {tot up }}^{-1}$ is the total probability of above-barrier neutron losses. The values $\tau_{\text {stor up }}$ and $\tau_{\text {tot up }}$ are measured experimentally with detector A without foil using an absorber for distinguishing the required part of the spectrum.

The scheme of measurement under consideration has not yet taken into account the fact that the time of collecting heated neutrons is finite. The error coefficient $f_{\text {up }}$ is to be calculated using Eq. (6),

$$
\begin{aligned}
f_{\text {up }}= & \left(\tau_{\text {stor below }}-\tau_{\text {tot up }}\right)^{-1} \times\left\{\tau_{\text {stor below }}\left[1-e^{-t_{\text {emp }} / \tau_{\text {stor below }}}\right]\right. \\
& \left.-\tau_{\text {tot up }}\left[1-e^{-t_{\text {emp }} / \tau_{\text {totup }}}\right]\right\}
\end{aligned}
$$

where $\tau_{\mathrm{emp}}$ is the time of measuring the number of heated neutrons.

As a result, one gets the following equation:

$$
W^{\text {up }}=\frac{N_{\text {up }}^{\text {det }}}{N_{0}^{\text {det }} \text { below }} \frac{P_{\text {below }}^{\text {det }}}{P_{\text {up }}^{\text {det }}} \frac{1}{\tau_{\text {stor below }}} \frac{1}{f_{\text {up }}} .
$$

Two measurements with gas and without are supposed to be made for gas heating probability:

$$
W_{\text {gas }}^{\text {up }}\left(n_{A}\right)=W^{\text {up }}\left(n_{A}\right)-W^{\text {up }}(0),
$$

thus, making allowance for UCN heating effect on the trap walls.

\section{RESULTS OF THE MAIN MEASUREMENTS}

A measuring cycle of the main measurements begins with filling the spectrometer with ultracold neutrons through the open entrance shutter (position 1 in Fig. 2). The filling duration is $100 \mathrm{~s}$. The count rate of detectors A and B during a measuring cycle is shown in Figs. 3 and 4. Detector A is placed behind a zirconium foil and measures the count rate
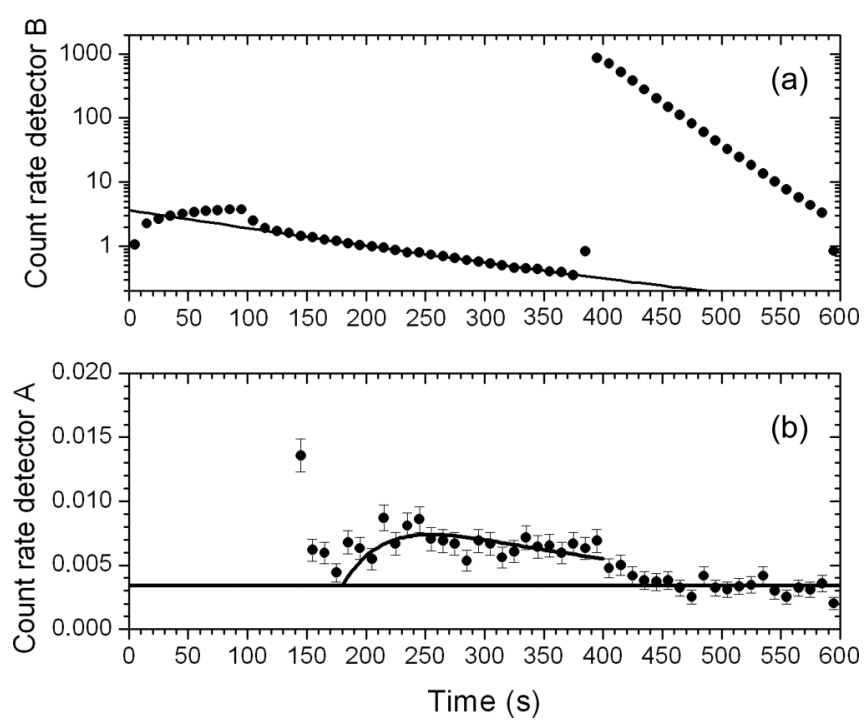

FIG. 3. Dependencies of count rate for detectors A and B on the measuring cycle with vacuum in the spectrometer. (a) Shows the count rate through the slit in the closed shutter for detector B within the time interval of $0-370 \mathrm{~s}$ and that in the open position within the time interval of 380-600 s. (b) Shows the count rate of up-scattered neutrons for detector A. The solid line corresponds to fitting according to Eq. (6). 

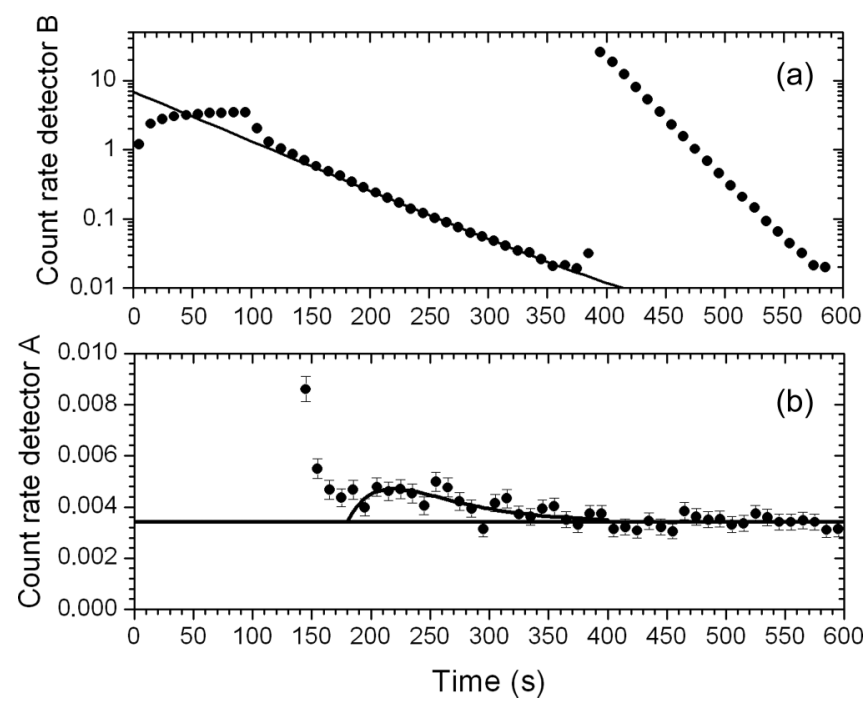

FIG. 4. Dependencies of count rate for detectors A and B on the time of measuring cycle when the spectrometer is filled with gas. (a) Shows the count rate through the slit in the closed shutter for detector B within the time interval of $0-370 \mathrm{~s}$ and in the open position within the time interval of 380-600 s. (b) Shows the count rate of up-scattered neutrons for detector A. The solid line corresponds to fitting according to Eq. (6).

of up-scattered neutrons. Detector B measures the count rate of UCN escaping through a small slit in the shutter (6). This count rate is proportional to UCN density in the spectrometer and practically does not affect the process of storing UCN. Figure 3 presents measurement data of the spectrometer under vacuum and Fig. 4 illustrates the case when the spectrometer is filled with gaseous He at a pressure of 3.5 mbar. The cited data are summarized and normalized according to the number of performed cycles. In total, there were 840 measuring cycles with vacuum in the spectrometer and 3510 cycles when the spectrometer was filled with He. Each cycle lasts for $10 \mathrm{~min}$.

An absorber is placed at a height of $85 \mathrm{~cm}$ from the spectrometer bottom during filling up and during the subsequent process referred to as cleaning of the UCN spectrum. After closing the entrance shutter, the cleaning of the UCN spectrum lasts for $80 \mathrm{~s}$ and is followed by lifting of the absorber with special gear to its maximum upper position (at a height of $211 \mathrm{~cm}$ from the spectrometer bottom). The aim of the UCN spectrum cleaning is to remove from the spectrometer the UCN with energies sufficient for transmission through zirconium foil in front of detector A (position 8 in Fig. 2).

The zirconium foil boundary energy is approximately equal to $76 \mathrm{neV}$. During cleaning of the UCN spectrum the absorber was placed at a height of $60 \mathrm{~cm}$ from the zirconium foil center and $85 \mathrm{~cm}$ from the spectrometer bottom. The absorber height and the time for spectrum cleaning are specially optimized.

After the absorber is lifted to its maximum upper position, neutrons transmitting through the zirconium foil are recorded for $200 \mathrm{~s}$. A typical peak (after $180 \mathrm{~s}$ ) is clearly seen in Fig. 3. This peak in count rate coincides with the moment of lifting the absorber and is accounted for by accumulation of the upscattered UCN in the spectrometer which were destructed up to $180 \mathrm{~s}$ in the absorber. In Fig. 4 such a peak is less noticeable because of a considerable decrease in the number of UCN in the spectrometer.

The recording of up-scattered neutrons is followed by opening the shutter (position 6 in Fig. 2) and all UCN remaining in the spectrometer are registered for $200 \mathrm{~s}$ with detector B (position 7 in Fig. 2). The number of UCN recorded during this process was used to control the stability of the main parameters of an experimental installation, namely, the time of UCN storage in the spectrometer, UCN flux from the source, proper operation of shutters and absorber.

The last $100 \mathrm{~s}$ of a measuring cycle were used to measure the background for detector $\mathrm{A}$. The background measurement with gas was $0.0034 \pm 0.0001 \mathrm{n} / \mathrm{s}$ and without gas it was $0.0031 \pm 0.0002 \mathrm{n} / \mathrm{s}$. The average ratio of the measured effect to the background in $200 \mathrm{~s}$ in measurements with gas is 0.2 and in measurements without gas is 1.1 .

As a result of the main measurements we obtained average values for the number of up-scattered neutrons in the spectrometer during measurements: $N_{\text {up }}^{\operatorname{det}}\left(n_{A}\right)=0.14 \pm 0.02 \mathrm{n} / \mathrm{s}$, with the spectrometer being at a pressure of $3.5 \mathrm{mbar} \mathrm{He}$ and $N_{\text {up }}^{\text {det }}(0)=0.71 \pm 0.06 \mathrm{n} / \mathrm{s}$ for measurements without gas.

Although the total number of up-scattered UCN in measurements with gas is much lower, the heating process of $\mathrm{UCN}$ on gas is readily observed, as one needs to compare the fraction of up-scattered neutrons to the quantity of sub-barrier neutrons. The presence of gas in the spectrometer considerably suppresses the quantity of sub-barrier neutrons, thus it is necessary to take the ratio of count rates of detectors $\mathrm{A}$ and $\mathrm{B}$ in measuring with gas and without. These ratios are presented in Fig. 5. It is to be noted that correction on alteration of registration efficiency $P_{\text {up }}^{\operatorname{det}}(0) / P_{\text {up }}^{\operatorname{det}}\left(n_{A}\right)$ was introduced for measuring with gas.

To summarize, we successfully demonstrated the process of UCN up-scattering on gas. The main problem is whether the values differ from admissible low-energy heating caused by conventional nuclear scattering. To solve this problem we made calibration measurements and calculated a low-energy up-scattering.

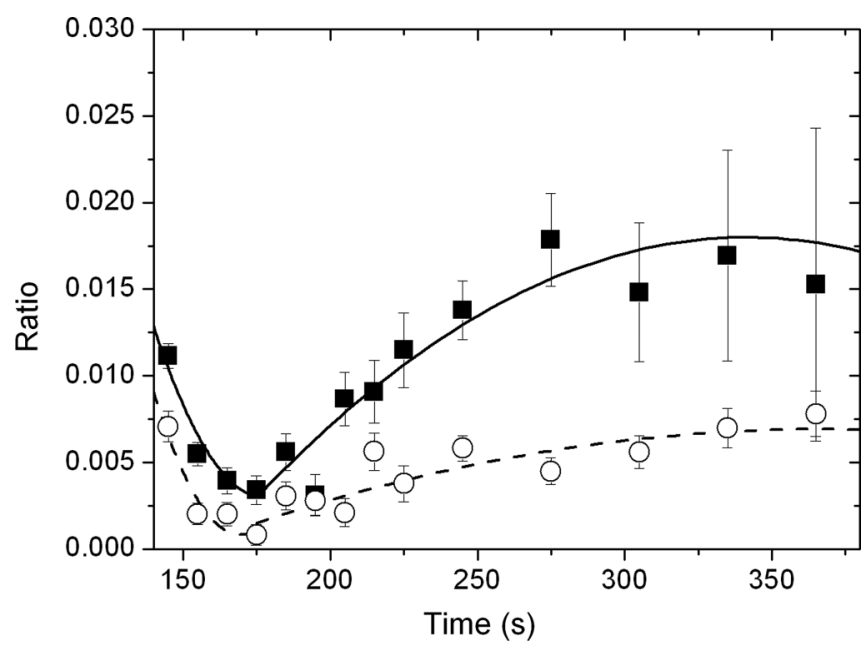

FIG. 5. Ratio of count rates of detectors A and B to the time of measuring cycle for the spectrometer filled with gas (squares, upper curve) and without gas (circles, lower curve). 


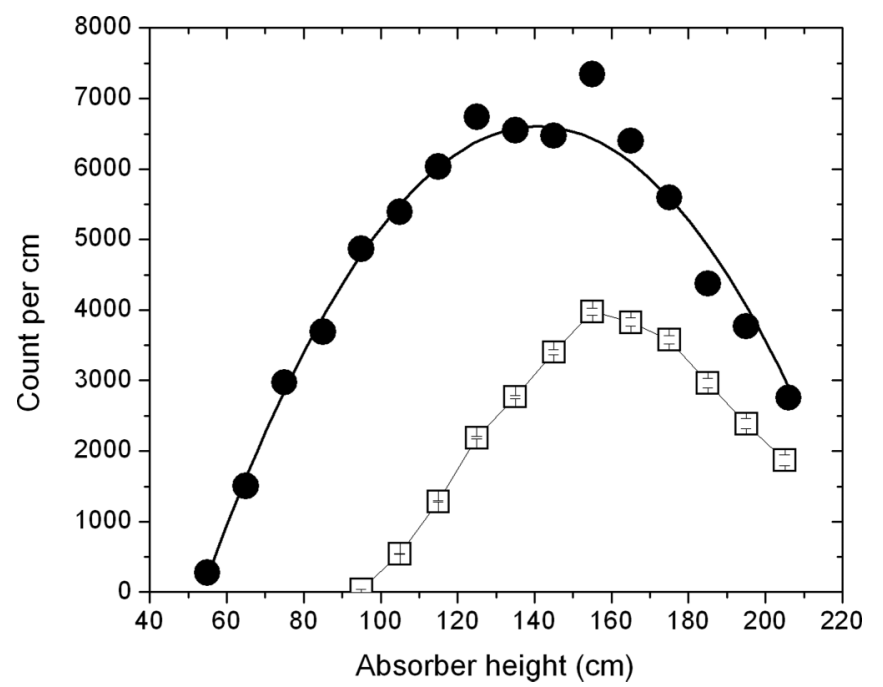

FIG. 6. Differential UCN spectra $N_{A}^{f}(E)$ for the spectrometer with foil (squares, low curve) and $N_{A}(E)$ for the spectrometer without foil (circles, upper curve).

\section{CALIBRATION MEASUREMENTS}

\section{A. Measuring factor $T_{\text {foil }}$}

Transmission coefficient $T_{\text {foil }}$ from Eq. (13) can be determined as the ratio of the counter rate of detector $\mathrm{A}$ in the scheme with foil $N_{A}^{f}$ and without foil $N_{A}$. Using a gravitational spectrometer one can obtain the energy dependence of the factor $T_{\text {foil }}(E)$.

Calibration measurements $T_{\text {foil }}(E)$ were made for UCN at different mean energy $E_{\mathrm{UCN}}$. To form various mean energies of UCN the spectrometer absorber during measurements is set at different heights. The results of the calibration measurements are given in Fig. 6. Differential spectra were obtained by differentiation of integral spectra for different absorber positions. The differential spectrum for the spectrometer with foil emerges at an energy of about $100 \mathrm{neV}$. UCN with lower energy are reflected from zirconium foil as the foil is located at a height of $25 \mathrm{~cm}$ from the spectrometer bottom, while the boundary energy of zirconium foil is $76 \mathrm{neV}$.

Calibration measurements $\tau_{\text {stor up }}(E)$ and $\tau_{\text {emp up }}(E)$ were carried out without foil for UCN with various energies.

Figure 7 demonstrates the ratio of differential spectra of UCN for the spectrometer with foil and without it $T_{\text {foil }}(E)=$ $N_{A}^{f}(E) / N_{A}(E)$ depending on UCN energy.

Further calculations use the average value of this ratio $\overline{T_{\text {foil }}}$ within interval $\left(E_{\mathrm{UCN}}^{\text {foil }}, E_{\mathrm{UCN}}^{\text {trap }}\right)$ from 95 to $205 \mathrm{neV}$, with $\overline{T_{\text {foil }}}$ being equal to 0.49 .

\section{B. Measuring UCN storage time and leakage time}

UCN storage time measurement in the trap $\tau_{\text {storbelow }}$ is a conventional procedure. It consists of measuring the number of UCN in the trap $N\left(t_{1}\right)$ at $t_{1}=80 \mathrm{~s}$ after closing the entrance valve (1) and the number of UCN in the trap $N\left(t_{2}\right)$ at $t_{2}=$ $280 \mathrm{~s}$. The storage time $\tau_{\text {storbelow }}$ is determined by $\tau_{\text {storbelow }}=$ $\ln \left(N\left(t_{1}\right) / N\left(t_{2}\right)\right) /\left(t_{2}-t_{1}\right)$. Selecting these particular times $t_{1}$ and $t_{2}$ is not incidental. During this time interval the up-

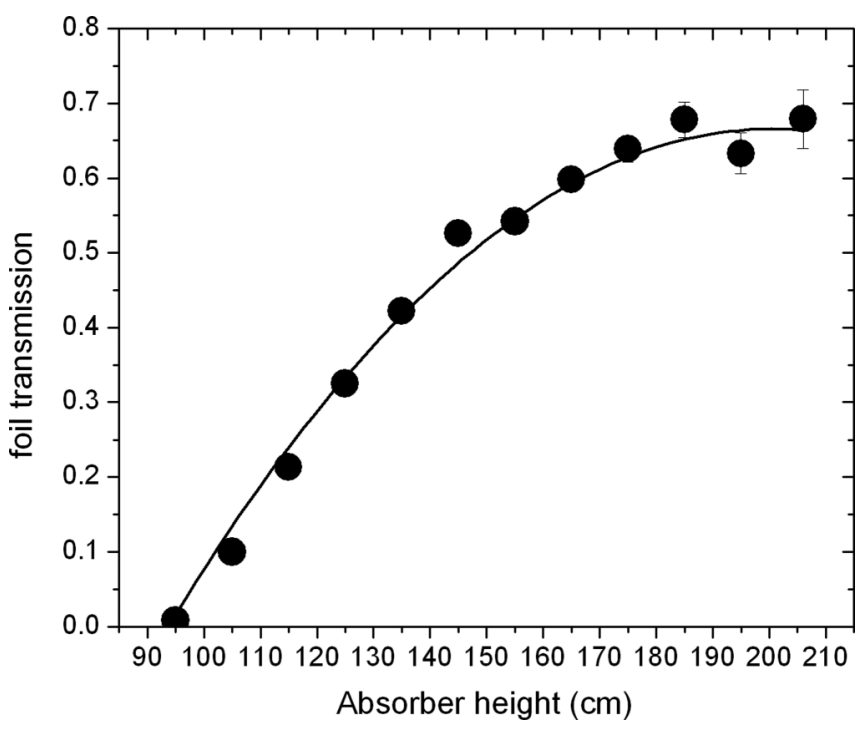

FIG. 7. Energy dependence of ratio of differential UCN spectra for the spectrometer with foil and without $T_{\text {foil }}(E)=N_{A}^{f}(E) / N_{A}(E)$.

scattered UCN are registered. As a result, the following value $\tau_{\text {stor below }}=162.6 \pm 0.4 \mathrm{~s}$ is obtained. The quantity $\tau_{\text {stor below }}$ for the case when the spectrometer is filled with gas was measured with detector B directly by storing sub-barrier UCN in a trap because a small leak valve closure (6) allows measuring UCN density decrease during storage. Storage time of UCN in the spectrometer filled with gas is $\tau_{\text {stor below }}^{\mathrm{He}}=56.3 \pm 1.0 \mathrm{~s}$.

Storage time of the spectrometer $\tau_{\text {stor up }}$ was determined as follows. Measurement of the UCN differential spectrum for the spectrometer without foil was made with a hold time of $50 \mathrm{~s}$ and $100 \mathrm{~s}$. Figure 6 shows the differential spectrum after $50 \mathrm{~s}$ of holding. Storage time of the spectrometer is calculated as

$$
\tau_{\text {stor up }}\left(E_{i}\right)=\ln \left(N_{50}^{*}\left(E_{i}\right) / N_{100}^{*}\left(E_{i}\right)\right) /(100-50),
$$

where $E_{i}$ is the mean UCN energy for $i$ energy interval, $N_{50}^{*}\left(E_{i}\right)$ and $N_{100}^{*}\left(E_{i}\right)$ are differential spectra with holding times $50 \mathrm{~s}$ and $100 \mathrm{~s}$, correspondingly. Total UCN losses in the spectrometer in configurations without foil $\tau_{\text {emp up }}^{-1}+\tau_{\text {stor up }}^{-1}$ and with foil $\tau_{\text {emp f }}^{-1}+\tau_{\text {stor up }}^{-1}$ are determined by least square fits of UCN leakage from the spectrometer onwards to detector A. We fit leakage curves of differential spectrum after $50 \mathrm{~s}$ holding time. Shown in Table I and in Fig. 8 are results of measuring storage time in the spectrometer $\tau_{\text {stor up }}$ and total UCN losses in the spectrometer without foil $1 /\left(\tau_{\text {emp up }}^{-1}+\tau_{\text {stor up }}^{-1}\right)$ and with foil $1 /\left(\tau_{\text {emp f }}^{-1}+\tau_{\text {stor up }}^{-1}\right)$ for different UCN energies.

An experimental transmission coefficient of above-barrier neutrons through zirconium foil (Fig. 7) and storage times of UCN (Fig. 8) were used for calculating the detection efficiency of above-barrier neutrons by the installation according to Eq. (14). The energy dependence of the detection efficiency of above-barrier neutrons by the installation is shown in Fig. 9. The decrease in detection efficiency at low energies is accounted for by the fact that above-barrier neutrons are not able to overcome the reflection potential of zirconium foil. The decrease at high energies is from deterioration of storage 
TABLE I. The storage time and total UCN losses in the spectrometer for different energies.

\begin{tabular}{lrcc}
\hline \hline$E_{\mathrm{UCN}}(\mathrm{neV})$ & \multicolumn{1}{c}{$\tau_{\text {stor up }}(\mathrm{s})$} & $1 /\left(\tau_{\text {emp up }}^{-1}+\tau_{\text {stor up }}^{-1}\right)(\mathrm{s}) 1 /\left(\tau_{\text {emp }}^{-1}+\tau_{\text {stor up }}^{-1}\right)(\mathrm{s})$ \\
\hline 205 & $50 \pm 4.1$ & $26.3 \pm 1.4$ & $33.7 \pm 1.9$ \\
195 & $69.8 \pm 6.0$ & $29.5 \pm 1.6$ & $36.7 \pm 1.6$ \\
185 & $71.3 \pm 5.1$ & $28.8 \pm 1.2$ & $41.1 \pm 1.5$ \\
175 & $73.1 \pm 3.8$ & $33.2 \pm 1.2$ & $44.3 \pm 1.2$ \\
165 & $91.2 \pm 5.1$ & $35.0 \pm 1.1$ & $50.7 \pm 1.2$ \\
155 & $91.8 \pm 3.9$ & $32.9 \pm 0.8$ & $54.1 \pm 1.2$ \\
145 & $95.6 \pm 4.4$ & $35.6 \pm 1.0$ & $58.4 \pm 1.2$ \\
135 & $103.3 \pm 4.2$ & $33.7 \pm 0.8$ & $64.5 \pm 1.0$ \\
125 & $105.5 \pm 4.4$ & $34.4 \pm 0.9$ & $69.7 \pm 1.0$ \\
115 & $106.4 \pm 3.9$ & $37.5 \pm 0.4$ & $79.8 \pm 1.2$ \\
105 & $118.7 \pm 4.9$ & $40.8 \pm 0.5$ & $83.3 \pm 1.4$ \\
\hline \hline
\end{tabular}

of above-barrier neutrons when their energy is close to the boundary energy of coating of the spectrometer.

\section{CALCULATION OF PROBABILITY OF UP-SCATTERING UCN ON GAS}

All values for Eq. (16) are included in Table II.

The probability value of UCN scattering with heating on gas $W_{\text {gas }}^{\text {up }}\left(n_{A}\right)$ is calculated according to Eq. (17) $W_{\text {gas }}^{\text {up }}\left(n_{A}\right)=$ $(3.1 \pm 1.0) \times 10^{-7} \mathrm{~s}^{-1}$. To be more exact, it is the probability of up-scattering on gas mixture in which He pressure is 3.49 mbar and in-leakage gas pressure is 0.03 mbar. Though the pressure of in-leakage gases in the course of measuring is small compared with the He pressure, their contribution to losses as well as UCN low-energy up-scattering turned out to be essential. UCN losses on $\mathrm{He}$ are $75.5 \%$ from the total losses on gas mixture, with UCN low-energy up-scattering on $\mathrm{He}$ being $46 \%$ from the total heating effect. This evidence resulted from a detailed measuring of losses and spectral measuring of

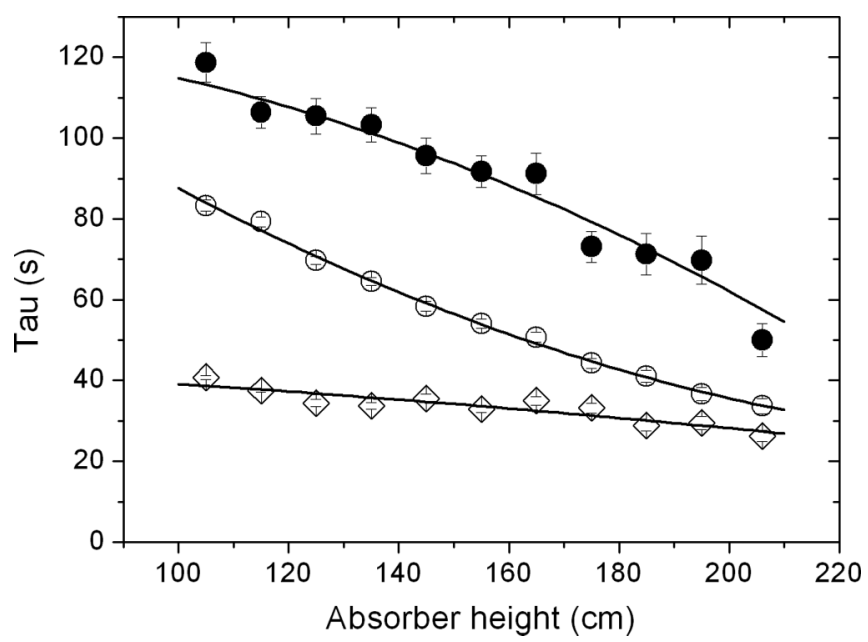

FIG. 8. Measuring results of storage time in the spectrometer $\tau_{\text {stor up }}$ (shown with circles), as well as the time of total UCN losses in the spectrometer in configurations with foil $1 /\left(\tau_{\text {emp f }}^{-1}+\tau_{\text {stor up }}^{-1}\right)$ (shown with hollow circles) and without foil $1 /\left(\tau_{\text {emp up }}^{-1}+\tau_{\text {stor up }}^{-1}\right)$ (shown with hollow rhombuses).

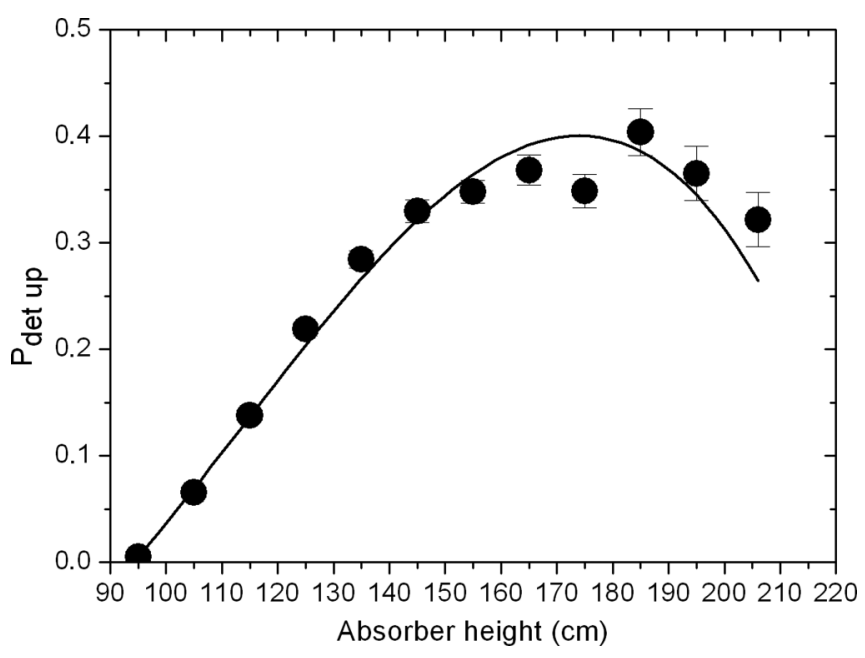

FIG. 9. Energy dependence of detection efficiency of abovebarrier neutrons, $P_{\text {up }}^{\text {det }}(E)=T_{\text {foil }}(E) \frac{\tau_{\text {empup }}^{-1}}{\tau_{\text {stor up }}^{-1}+\tau_{\text {emp up }}^{-1}}$.

in-leakage gas composition. In particular, UCN losses on the spectrometer walls are $(162 \mathrm{~s})^{-1}$, losses on gas mixture being $(86.1 \mathrm{~s})^{-1}$, and UCN losses only on He being $(114 \mathrm{~s})^{-1}$.

It should be taken into consideration that the probability of low-energy up-scattering on gases is proportional to the multiplier $(M+1)^{2} / 4 M$. Consequently, special attention should be given to the gas mixture composition in the spectrometer during measurements. The composition of in-leakage gases in the spectrometer after shutting off the evacuation pump is measured with a mass spectrometer made by Hiden Analytical. Measurement of gas composition is made at pressure in the range of $2 \times 10^{-5} \div 2 \times 10^{-4}$ mbar. Water, hydrogen, and air are basic constituents of the in-leakage gas mixture. Gas mixture composition and gas properties are given in Table III.

Contributions to heating for helium and admixtures are distributed as $46 \%$ and $54 \%$, correspondingly, as seen in the far right column of Table III. Thus, the effect of low-energy up-scattering on helium is evaluated as $1.4 \times 10^{-7} \mathrm{~s}^{-1}$. As for errors, we choose their maximum value $1.0 \times 10^{-7} \mathrm{~s}^{-1}$ for reliability. As a result, one obtains the following equation:

$$
W_{\mathrm{He}}^{\mathrm{up}}\left(n_{A}\right)=(1.4 \pm 1.0) \times 10^{-7} \mathrm{~s}^{-1}
$$

\section{ANALYSIS OF THE OBTAINED RESULTS ON EXISTENCE OF LONG-RANGE FORCES}

The obtained results can be used for setting constraints on values $g^{2}$ and $\lambda$ for hypothetical long-range interaction. While taking into account long-range forces it is necessary to make the following calculations. In the Born approximation the total amplitude of neutron scattering on gas atoms of mass $M m_{n}$ can be written as follows:

$$
f_{\text {total }}(q)=-b_{\text {free_nucl }}+\frac{\mp g_{ \pm}^{2} M^{2}}{2 \pi(M+1) \lambda_{C}} \frac{\lambda^{2}}{(q \lambda)^{2}+1} .
$$

Contribution in the scattering amplitude is made by shortrange nuclear interaction with amplitude corresponding to $f_{\text {free_nucl }}=-b_{\text {free_nucl }}\left(b_{\text {free_nucl- }}\right.$ nuclear scattering length of 
TABLE II. The values used in Eq. (16) for measurements with helium (at pressure of 3.5 mbar) and with vacuum.

\begin{tabular}{lcccccrl}
\hline \hline$n_{A}$ & $N_{\text {Obelow }}^{\text {det }}$ & $N_{\text {up }}^{\text {det }}$ & $P_{\text {up }}^{\text {det }}$ & $P_{\text {below }}^{\text {det }}$ & $\tau_{\text {stor }}^{\text {below }}(\mathrm{s})$ & $f_{\text {up }}$ & $W^{\text {up }}\left(n_{A}\right)\left(\mathrm{s}^{-1}\right)$ \\
\hline 3.5 & $8900 \pm 90$ & $0.14 \pm 0.02$ & $0.190 \pm 0.008$ & $0.393 \pm 0.004$ & $56.3 \pm 1.0$ & $0.932 \pm 0.004$ & $(6.2 \pm 0.9) \times 10^{-07}$ \\
0 & $51360 \pm 230$ & $0.71 \pm 0.06$ & $0.31 \pm 0.01$ & $0.648 \pm 0.005$ & $162.6 \pm 0.4$ & $0.570 \pm 0.006$ & $(3.1 \pm 0.3) \times 10^{-07}$ \\
\hline \hline
\end{tabular}

neutron on a free atom), with the second term in Eq. (20) describing the contribution of long-range forces in Eq. (3).

If the velocity of an ultracold neutron before its collision with a gas atom is much lower than an average thermal velocity of atoms $\overline{V_{A}}=4\left(k_{B} T / 2 \pi m_{n} M\right)^{1 / 2}$, then the square of momentum $q$ is related to the energy $\varepsilon$ transmitted to a neutron, $q^{2}=2 \varepsilon m_{n} / \hbar^{2}$. Assuming a neutron to be at rest before its collision with an atom, one obtains the equation for the element of solid angle $d \Omega$, into which UCN can be scattered depending on the incident atom energy $-E_{A}$ and its mass,

$$
d \Omega=\frac{\pi(M+1)^{2}}{M} \frac{d \varepsilon}{E_{A}} .
$$

Thus, the equation for the differential cross section $d \sigma(\varepsilon)=$ $\left|f_{\text {total }}(\varepsilon)\right|^{2} d \Omega$ will be equal to

$$
\begin{aligned}
d \sigma(\varepsilon)= & \pi \frac{(M+1)^{2}}{M}\left(b_{\text {free_nucl }}^{2} \pm \frac{g_{ \pm}^{2} M^{2}}{\pi(M+1)} \frac{b_{\text {free_nucl }}}{\lambda_{C}}\right. \\
& \left.\times \frac{\lambda^{2}}{2 m_{n} \varepsilon \lambda^{2} / \hbar^{2}+1}+f_{\text {long range }}^{2}\right) \frac{d \varepsilon}{E_{A}}
\end{aligned}
$$

where $f_{\text {long range }}$ is a scattering amplitude from long-range forces [Eq. (3)].

The probability of conventional nuclear UCN up-scattering from 0 to $E_{\mathrm{UCN}}^{\text {foil }}$ into the range from $E_{\mathrm{UCN}}^{\text {foil }}$ to $E_{\mathrm{UCN}}^{\text {trap }}$ can be calculated as a product of the atomic beam scattering cross section (see formula (22) from [23]):

$$
\begin{aligned}
& W_{\text {nucl }}\left(n_{A}\right) \\
& =\int_{E_{\mathrm{UCN}}^{\text {foil }}}^{E_{\mathrm{UCN}}^{\mathrm{trap}}} d \sigma(\varepsilon) \int_{\varepsilon \frac{(M+1)^{2}}{4 M}}^{\infty} d \Phi\left(E_{A}\right) \\
& =\frac{(M+1)^{2}}{4 M} n_{A} 4 \pi b_{\text {free_nucl }}^{2} \frac{\bar{V}_{A}}{k_{B} T} \int_{E_{\mathrm{UCN}}^{\mathrm{foil}}}^{E_{\mathrm{UCN}}^{\mathrm{tra}}} e^{-\varepsilon \frac{(M+1)^{2}}{4 M} / k_{B} T} d \varepsilon \\
& =n_{A} \bar{V}_{A} \frac{\left(E_{\mathrm{UCN}}^{\mathrm{trap}}-E_{\mathrm{UCN}}^{\mathrm{foil}}\right)}{k_{B} T} \frac{(M+1)^{2}}{M} 4 \pi b_{\text {free_nucl }}^{2}
\end{aligned}
$$

TABLE III. The gas mixture composition and contributions to the low-energy up-scattering.

\begin{tabular}{llccccc}
\hline \hline Gas & $p$ (mbar) & $(p \tau)($ mbar s) & $\frac{p}{(p \tau)}\left(\mathrm{s}^{-1}\right)$ & $M$ & $\frac{(M+1)^{2}}{4 M}$ & $\frac{p}{(p \tau)} \frac{(M+1)^{2}}{4 M}\left(\mathrm{~s}^{-1}\right)$ \\
\hline $\mathrm{He}$ & 3.49 & 418 & 0.0083 & 4 & 1.56 & 0.013 \\
$\mathrm{H}_{2} \mathrm{O}$ & 0.015 & 6.7 & 0.0022 & 18 & 5 & 0.011 \\
$\mathrm{H}_{2}$ & 0.008 & 4.9 & 0.0016 & 2 & 1.13 & 0.002 \\
$\mathrm{Air}$ & 0.007 & 24.7 & 0.0003 & 29 & 7.79 & 0.002 \\
& & & & & $\mathbf{0 . 0 2 8}$ \\
\hline \hline
\end{tabular}

This calculation is based on the assumption that UCN are at rest before interacting with $\mathrm{He}$ atoms. The calculation with the Maxwell UCN velocities spectrum provides the same result.

Values of variables in Eq. (23) are given below:

$$
\begin{aligned}
n_{A} & =2.687 \times 10^{16} \times 273 / 293 \times 3.49=8.737 \times 10^{16}, \\
\bar{V}_{A} & =1.240 \times 10^{5} \mathrm{~cm}^{2}, \\
E_{\mathrm{UCN}}^{\text {trap }} & =2.42 \times 10^{-7} \mathrm{eV}(6.8 \mathrm{~m} / \mathrm{s}) ; \\
E_{\mathrm{UCN}}^{\text {foil }}= & 0.756 \times 10^{-7} \mathrm{eV}(3.8 \mathrm{~m} / \mathrm{s}), \\
4 \pi b_{\text {free_nucl }}^{2}= & 0.76 \times 10^{-24} \mathrm{~cm}^{2}, \\
k_{B} T= & 0.0252 \mathrm{eV} .
\end{aligned}
$$

Calculation with these magnitudes provides the probability value of UCN transfer from the energy range lower than $E_{\mathrm{UCN}}^{\text {foil }}$ into the energy range $E_{\mathrm{UCN}}^{\text {foil }}<\varepsilon<E_{\mathrm{UCN}}^{\text {trap }}-W_{\text {nucl }}=$ $0.8495 \times 10^{-7} \mathrm{~s}^{-1}$ (for $4 \pi b_{\text {free_nucl }}^{2}=0.76 \times 10^{-24} \mathrm{~cm}^{2}$ ). The experimentally obtained magnitude for this value is Eq. (19) $(1.4 \pm 1.0) \times 10^{-7} \mathrm{~s}^{-1}$. We thus observe no evidence of excess over the low-heating probability. This result, however, does not allow imposing new constraints on the possible contribution of long-range forces. One should make calculations with a similar expression for the probability of UCN low-energy heating from long-range forces by the following equation with an additional multiplier in square brackets,

$$
\begin{aligned}
W_{\mathrm{at}}^{\text {low }}\left(n_{A}\right)= & n_{A} \bar{V}_{A} \frac{\left(E_{\mathrm{UCN}}^{\mathrm{trap}}-E_{\mathrm{UCN}}^{\mathrm{foil}}\right)}{k_{B} T} \frac{(M+1)^{2}}{M} 4 \pi b_{\text {free_nucl }}^{2} \\
& \times\left[1 \pm \frac{1}{2} J_{1}\left(g_{ \pm}^{2}, \lambda\right)\right]^{2}
\end{aligned}
$$

where

$$
J_{1}\left(g_{ \pm}^{2}, \lambda\right)=\frac{g_{ \pm}^{2} M^{2}}{\pi(M+1)} \frac{1}{b_{\text {free_nucl } \bar{\lambda}_{C}}} \frac{\lambda^{2}}{\lambda^{2} / \lambda_{\mathrm{UCN}}^{2}+1} . \quad \text { Value }
$$
$\overline{\bar{\lambda}}_{\mathrm{UCN}}$ is equal to the de Broglie wavelength of a neutron $\left(\bar{\lambda}_{\mathrm{UCN}}^{2}=\hbar^{2} / 2 m_{n} \bar{\varepsilon}\right)$ moving with energy $\bar{\varepsilon}=\left(E_{\mathrm{UCN}}^{\text {trap }}+E_{\mathrm{UCN}}^{\text {foil }}\right) / 2$.

Equating $W_{\text {at }}^{\text {low }}\left(n_{A}\right)$ from Eq. (24) to the experimental magnitude obtained in this work $\left(W_{\text {gas }}^{\text {up }}\left(n_{A}\right)^{\exp }=(1.4 \pm 1.0) \times\right.$ $\left.10^{-7} \mathrm{~s}^{-1}\right)$ and taking its ratio to the corresponding probability calculated with account for nuclear scattering only [Eq. (23)], we get the following equality:

$$
\frac{W_{\text {at }}^{\text {low }}\left(n_{A}\right)}{W_{\text {nucl }}\left(n_{A}\right)}=\left[1 \pm \frac{1}{2} J_{1}\left(g_{ \pm}^{2}, \lambda\right)\right]^{2}=1.65 \pm 1.2 .
$$

It is seen that, unfortunately, the accuracy attained in our measurements is not sufficient to unambiguously choose the sign in the square brackets and thus to determine the sign of the potential $\varphi$ in Eq. (2). Here and further we will choose the sign in square brackets so that the value $g_{ \pm}^{2}$ derived from this inequality would be the lowest. The following inequality is derived from Eq. (25) for function $J_{1}: 0<J_{1}\left(g_{+}^{2}, \lambda\right)<1.8$ 


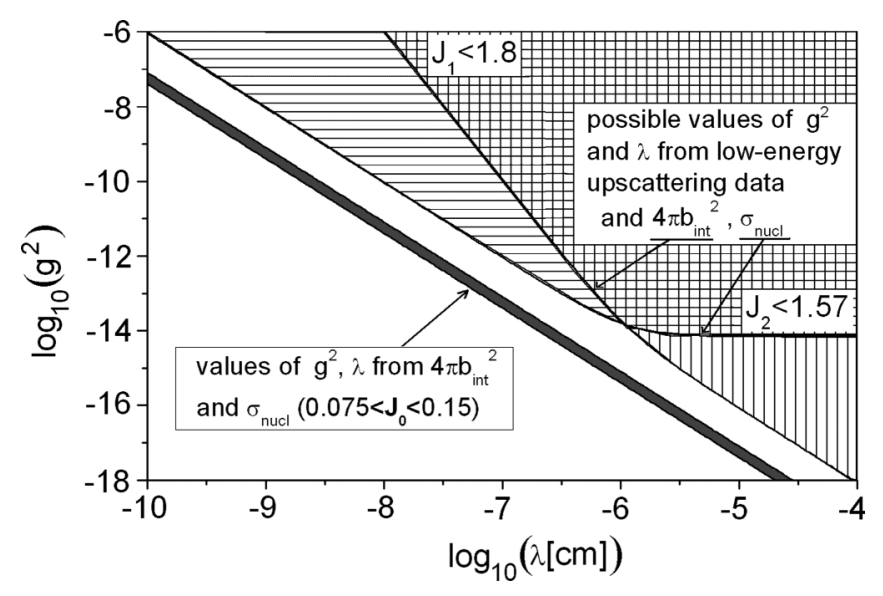

FIG. 10. The exclusion (shaded) and allowed (gray) areas of values $g^{2}$ and $\lambda$ from different experimental data.

(90\% confidence level). By solving this inequality one finds the range of allowed values $g_{+}^{2}$ and $\lambda$, given in Fig. 10 .

To distinguish the contribution of long-range forces in UCN scattering one can use the measurement results on coherent scattering length made with neutron interferometers. In Eq. (20) by subtracting and adding the contribution of longrange forces in scattering at zero angle one can distinguish a coherent scattering amplitude measured with interferometer $-b_{\text {free_int }}$, and finally derive a formula for the total scattering amplitude,

$$
f_{\text {total }}(\varepsilon)=-\left(b_{\text {free_int }} \mp \frac{g_{ \pm}^{2} M^{2}}{2 \pi(M+1)} \frac{\lambda^{2}}{\lambda_{C}}\left(\frac{2 m_{n} \varepsilon \lambda^{2} / \hbar^{2}}{2 m_{n} \varepsilon \lambda^{2} / \hbar^{2}+1}\right)\right) .
$$

In this case a similar expression for the probability of UCN low-energy heating using neutron interferometer data and taking into account long-range forces is presented by the following formula:

$$
\begin{aligned}
W_{\mathrm{at}}^{\mathrm{low}}\left(n_{A}\right)= & n_{A} \bar{V}_{A} \frac{\left(E_{\mathrm{UCN}}^{\mathrm{trap}}-E_{\mathrm{UCN}}^{\mathrm{foil}}\right)}{k_{B} T} \frac{(M+1)^{2}}{M} 4 \pi b_{\text {free_int }}^{2} \\
& \times\left[1 \mp \frac{1}{2} J_{2}\left(g_{ \pm}^{2}, \lambda\right)\right]^{2}
\end{aligned}
$$

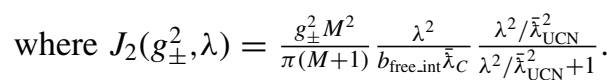

From comparison of Eqs. (23) and (27), one can see that (given that $4 \pi b_{\text {free_int }}^{2}=0.855 \times 10^{-24} \mathrm{~cm}^{2}$ and $\left.4 \pi b_{\text {freennucl }}^{2}=0.76 \times 10^{-24} \mathrm{~cm}^{2}\right)$ the value of the factor before the square brackets in Eq. (27) $\left(W_{\text {int }}\right)$ is equal to $W_{\text {int }}=0.9557 \times 10^{-7} \mathrm{~s}^{-1}$. To obtain the constraint on value $g^{2}(\lambda)$, one should compare this calculated magnitude with an experimental one. Equating $W_{\mathrm{at}}^{\text {low }}\left(n_{A}\right)$ from Eq. (27) to an experimental magnitude $W_{\text {gas }}^{\text {up }}\left(n_{A}\right)^{\exp }=(1.4 \pm 1.0) \times 10^{-7} \mathrm{~s}^{-1}$ and dividing it by $W_{\text {int }}=0.9557 \times 10^{-7} \mathrm{~s}^{-1}$, one obtains

$$
\frac{W_{\mathrm{at}}^{\text {low }}\left(n_{A}\right)}{W_{\mathrm{int}}\left(n_{A}\right)}=\left[1 \mp \frac{1}{2} J_{2}\left(g_{ \pm}^{2}, \lambda\right)\right]^{2}=1.46 \pm 1.05 .
$$

Having chosen the lower sign one gets the following inequality: $0<J_{2}\left(g_{-}^{2}, \lambda\right)<1.57$ (90\% confidence level). Having solved this inequality one finds the range of allowed values $g_{-}^{2}$ and $\lambda$, given in Fig. 10.

Finally, we can obtain the expression for comparing the coherent scattering cross section $4 \pi b_{\text {free_int }}^{2}=0.852(16) \times 10^{-24}$ $\mathrm{cm}^{2}$, calculated using coherent scattering length obtained by the neutron interferometer technique and cross section of nuclear scattering $\sigma_{\text {free_nucl }}(\mathrm{He})=(0.76 \pm 0.01) \times 10^{-24} \mathrm{~cm}^{2}$ measured by the method of neutron transmission and by UCN storage time in He gas $(0.740 \pm 0.006) \times 10^{-24} \mathrm{~cm}^{2}$. Because the last two results practically coincide we restrict ourselves with comparison $\sigma_{\text {free_nucl }}(\mathrm{He})$ and $4 \pi b_{\text {free_int }}^{2}$ [23]:

$$
\frac{\sigma_{\text {free_nucl }(\mathrm{He})}}{4 \pi b_{\text {free_int }}^{2}}=\left[1 \mp \frac{1}{2} J_{0}\left(g_{ \pm}^{2}, \lambda\right)\right]^{2},
$$

where $J_{0}\left(g_{ \pm}^{2}, \lambda\right)=\frac{g_{ \pm}^{2} M^{2}}{\pi(M+1)} \frac{\lambda^{2}}{b_{\text {free.jint } \lambda_{C}}}$.

Using available experimental data we have the following for comparing the nuclear scattering data and those of the neutron interferometer:

$$
\left[1 \mp \frac{1}{2} J_{0}\left(g_{ \pm}^{2}, \lambda\right)\right]^{2}=0.888 \pm 0.023
$$

One gets the following constraint $0.85<[1 \mp$ $\left.\frac{1}{2} J_{0}\left(g_{ \pm}^{2}, \lambda\right)\right]^{2}<0.926$ with a $90 \%$ confidence interval. It can be seen from this inequality that in the given case one should choose the upper sign corresponding to the case of repulsion. The following inequality with an allowance for the sign is derived:

$$
0.075<J_{0}\left(g_{+}^{2}, \lambda\right)<0.15
$$

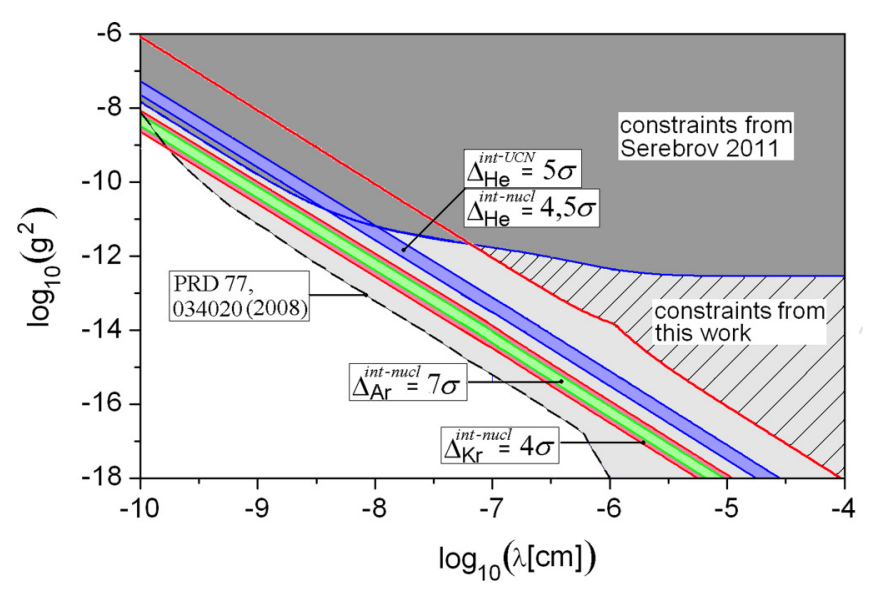

FIG. 11. (Color online) The dark shaded area corresponds to constraints for $g^{2}$ and $\lambda$ from the paper by Serebrov [23] (a comparison of the scattering cross section, evaluated from the storage time of $\mathrm{UCN}$ in $\mathrm{He}$ gas and the nuclear scattering cross section, measured by the neutron transmission). The dotted line shows constraints from Ref. [9] obtained as the best limit for heavy atoms ( $\mathrm{Pb}, \mathrm{Bi})$. The upper inclined area $\Delta_{\mathrm{He}}^{\mathrm{int}-\mathrm{UCN}}$ corresponds to a comparison of the scattering cross section, evaluated from the storage time of UCN, and the interferometer method [24] for He. The other inclined areas $\Delta_{\mathrm{He}}^{\mathrm{int} n u c l}$, $\Delta_{\mathrm{Ar}}^{\mathrm{int} \text {-nucl }}, \Delta_{\mathrm{Kr}}^{\mathrm{int} \text {-nucl }}$ corresponds to the values $g^{2}, \lambda$ from comparison of the scattering cross sections measured by the transmission method [25] and by means of the interferometer [24] for He, Ar, and $\mathrm{Kr}$. The red line corresponds to constraints for $g^{2}$ and $\lambda$ obtained in the present work. 
The range of allowed values $g_{+}^{2}, \lambda$, obtained from solution (30), is shown in Fig. 10.

Unfortunately, accuracy of the performed experiment proved to be insufficient to close or to confirm the range of values $g_{+}^{2}, \lambda$, which originated from the difference $4 \pi b^{2}(\mathrm{He})_{\text {free_int }}-\sigma_{\text {free_nucl }}(\mathrm{He})$ and differing from zero at the level of five standard deviations. However, these measurements of low-energy heating allow us to set new constraints on parameters $g_{+}^{2}, \lambda$ as compared with the work [23], which used the data on the nuclear scattering cross section obtained by the neutron transmission method as well as by that of measuring UCN storage time in He. Figure 11 makes a comparison between constraints from the work in [23] and those from the present work.

In addition to the range of values $g^{2}, \lambda$ from discrepancy between data on scattering and on interferometer data $\Delta^{\text {int-nucl }}$ at the level of five standard deviations for helium, Fig. 11 also shows a similar discrepancy for $\operatorname{Ar}(7 \sigma)$ and $\mathrm{Kr}(4 \sigma)$, that corresponds to $18 \%$ and $11 \%$, respectively. The dotted line shows constraints from Ref. [9] obtained as the best limit for heavy atoms. Finally, the hatched area corresponds to new constraints on values $g^{2}, \lambda$ from measurements of the lowenergy up-scattering, which were carried out in this work.

\section{CONCLUSIONS}

The attained accuracy of measuring the low-energy scattering cross section does not permit us to make an unambiguous conclusion on the presence of additional interactions caused by long-range forces between a neutron and $\mathrm{He}$ atom. To achieve the required accuracy with the help of the suggested methods it is necessary to considerably increase the UCN density. On the other hand, new measurements of $4 \pi b_{\text {free_int with a }}^{2}$ detailed analysis of possible systematic errors are capable of solving the problem of discrepancy between the scattering cross section $4 \pi b_{\text {free_int }}^{2}$, calculated through coherent scattering length measured with a neutron interferometer and nuclear scattering cross section measured by UCN storage time in helium. The positive conclusion of the present research is setting new constraints on the parameters $g^{2}$ and $\lambda$. These constraints become possible because of the application of a new experimental method.

In conclusion, it should be noted that the most probable reason for the discrepancy between $4 \pi b^{2}(\mathrm{He})_{\text {free_int }}$ and $\sigma_{\text {free_nucl }}(\mathrm{He})$ (that amounts to $10 \%$ ) is systematic errors. It is worth mentioning that a similar discrepancy between $4 \pi b_{\text {free_int }}^{2}$ and $\sigma_{\text {free_nucl }}$ was also discovered in measurements on argon and krypton, $18 \%$ and $11 \%$, respectively. Nevertheless, for lead and bismuth the discrepancy amounts to only $1 \%$ and moreover possesses an opposite sign relative to that for helium. So this discrepancy can be explained by the neutron-electron interaction. Systematic errors can be assumed to be associated with experiments on gases. A scientific team at NIST is planning to test some systematic errors and remeasure neutron the coherent scattering length of ${ }^{4} \mathrm{He}$ using the neutron interferometer technique.

\section{ACKNOWLEDGMENTS}

This work was supported by the Russian Foundation for Basic Research (Projects No. 08-02-01052a, No. 1002-00217a, and No. 10-02-00224a) and by the Ministry of Education and Science of the Russian Federation (Contracts No. 02.740.11.0532 and No. 14.740.11.0083). We would like to thank T. Brenner for intensive and very helpful assistance during the experiment.
[1] E. G. Floratos and G. K. Leontaris, Phys. Lett. B 465, 95 (1999).

[2] A. Kehagias and K. Sfetsos, Phys. Lett. B 472, 39 (2000).

[3] I. Antoniadis, S. Dimopoulos, and G. Dvali, Nucl. Phys. B 516, 70 (1998).

[4] S. S. Gubser and J. Khoury, Phys. Rev. D 70, 104001 (2004).

[5] A. Upadhye, S. S. Gubser, and J. Khoury, Phys. Rev. D 74, 104024 (2006).

[6] E. G. Adelberger, B. R. Heckel, and A. E. Nelson, Ann. Rev. Nucl. Part. Sci. 53, 77 (2003).

[7] E. G. Adelberger, J. H. Gundlach, B. R. Heckel, S. Hoedl, and S. Schlamminger, Prog. Part. Nucl. Phys. 62, 102 (2009).

[8] Yu. Kamyshkov, J. Tithof, and M. Vysotsky, Phys. Rev. D 78, 114029 (2008).

[9] V. V. Nesvizhevsky, G. Pignol, and K. V. Protasov, Phys. Rev. D 77, 034020 (2008).

[10] Yu. N. Pokotilovski, Phys. Atom. Nucl. 69, 924 (2006).

[11] D. J. Kapner, T. S. Cook, E. G. Adelberger, J. H. Gundlach, B. R. Heckel, C. D. Hoyle, and H. E. Swanson, Phys. Rev. Lett. 98, 021101 (2007).

[12] A. A. Geraci, S. J. Smullin, D. M. Weld, J. Chiaverini, and A. Kapitulnik, Phys. Rev. D 78, 022002 (2008).

[13] J. C. Long, H. W. Chan, A. B. Churnside, E. A. Gulbis, M. C. M. Varney, and J. C. Price, Nature (London) 421, 922 (2003).
[14] B. W. Harris, F. Chen, and U. Mohideen, Phys. Rev. A 62, 052109 (2000).

[15] E. Fischbach, D. E. Krause, V. M. Mostepanenko, and M. Novello, Phys. Rev. D 64, 075010 (2001).

[16] R. S. Decca, D. Lopez, H. B. Chan, E. Fischbach, D. E. Krause, and C. Jamell, Phys. Rev. Lett. 94, 240401 (2005).

[17] S. K. Lamoreaux, Phys. Rev. Lett. 78, 5 (1997).

[18] M. Bordag, B. Geyer, G. L. Klimchitskaya, and V. M. Mostepanenko, Phys. Rev. D 58, 075003 (1998).

[19] J. Chiaverini, S. J. Smullin, A. A. Geraci, D. M. Weld, and A. Kapitulnik, Phys. Rev. Lett. 90, 151101 (2003).

[20] D. Hoyle, U. Schmidt, B. R. Heckel, E. G. Adelberger, J. H. Gundlach, D. J. Kapner, and H. E. Swanson, Phys. Rev. Lett. 86, 1418 (2001).

[21] G. L. Greene and V. Gudkov, Phys. Rev. C 75, 015501 (2007).

[22] V. F. Sears, Phys. Rep. 141, 281 (1986).

[23] A. P. Serebrov, O. M. Zherebtsov, S. V. Sbitnev, V. E. Varlamov, A. V. Vassiljev, and M. S. Lasakov, Phys. Rev. C 84, 044001 (2011).

[24] H. Kaiser, H. Rauch, G. Badurek, W. Bauspiess, and U. Bonse, Z. Physik A 291, 231 (1979). 
[25] D. C. Rorer, B. M. Ecker, and R. Ö. Akyüz, Nucl. Phys. A 133, 410 (1969).

[26] S. F. Mughabghab, M. Divadeenam, and N. E. Holden, Neutron Cross Sections Series, Vol.1, Part A (Academic Press, New York, 1981).
[27] A. P. Serebrov, P. Geltenbort, O. M. Zherebtsov, S. V. Sbitnev, V. E. Varlamov, A. V. Vassiljev, M. S. Lasakov, I. A. Krasnoschekova, and S. N. Ivanov, JETP Lett. 97, 676 (2013).

[28] www.ncnr.nist.gov/resources/n-lengths/; www.ati.ac.at/ neutropt/scattering/Scattering_lengths_table_20010419.pdf 